\title{
An experimental and theoretical study of ring closing dynamics in $\mathrm{HN}_{3}$
}

\author{
Jianyang Zhang, ${ }^{a b}$ Peng Zhang, ${ }^{c}$ Yuan Chen, ${ }^{a}$ Kaijun Yuan, ${ }^{a}$ Steven A. Harich, ${ }^{a}$ \\ Xiuyan Wang, ${ }^{a b}$ Zhi Wang, ${ }^{c}$ Xueming Yang, ${ }^{a}$ Keiji Morokuma $^{c}$ and \\ Alec M. Wodtke $* d$
}

Received 16th January 2006, Accepted 17th February 2006

First published as an Advance Article on the web 1st March 2006

DOI: $10.1039 /$ b600599c

\begin{abstract}
We report on an $\mathrm{H}(\mathrm{D})$-atom Rydberg tagging experiment for $\mathrm{H}(\mathrm{D}) \mathrm{N}_{3}$ photolysis providing detailed dynamical information on the wavelength dependence of the $\mathrm{H}(\mathrm{D})+\mathrm{N}_{3}$ channel. We observe subtle yet striking changes in the photochemical dynamics as the photolysis energy passes through $\sim 5.6 \mathrm{eV}$. In addition to producing linear azide with an average of $\sim 40 \%$ of available energy appearing as translation, a second $\mathrm{H}(\mathrm{D})$-atom producing channel grows in above this energy releasing only about $15 \%$. An observed (inverse) isotope effect suggests that statistical decomposition on $\mathrm{S}_{0}$ is unimportant. High level ab initio quantum chemical calculations reveal a transition state to cyclization of the $\mathrm{N}_{3}$ moiety in $\mathrm{H}(\mathrm{D}) \mathrm{N}_{3}$ on the first excited singlet $\left(\mathrm{S}_{1}\right)$ surface that is close in energy to the experimentally observed threshold energy for this "slow channel". Furthermore, the translational energy release of the "slow channel" is energetically consistent with cyclic- $\mathrm{N}_{3}$ formation. This work provides the clearest presently available insights into how ring closure can occur in azide photochemistry.
\end{abstract}

\section{Introduction}

While a large number of all-nitrogen species have been predicted theoretically, ${ }^{1-6}$ there are to date only five allotropes of nitrogen that have been identified experimentally, ${ }^{7,8}$ three of which are $\mathrm{N}_{2}, \mathrm{~N}_{3}$ and $\mathrm{N}_{3}{ }^{-}$. One of the fundamental barriers to exploring the full diversity of all-nitrogen molecules is their near pathological resistance to ring formation. Experimental schemes that could demonstrate ring formation would provide new possibilities to the expansion of this intriguing field of chemistry. For some time, one of us (AMW) has proposed that cyclic-N species might be produced from suitable photochemical precursors. Several recent experiments ${ }^{9-14}$ provide evidence that the UV photolysis of $\mathrm{ClN}_{3}$ leads efficiently to the simplest cyclic all-nitrogen allotrope, cyclic- $\mathrm{N}_{3} \cdot{ }^{15-18}$

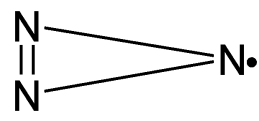

These results stand, however, in stark contrast to the paucity of theoretical studies on $\mathrm{ClN}_{3}$ photochemistry and consequently, the photochemical mechanism remains unclear as to which azides may undergo photochemical ring-closure. In the hopes of perhaps finding another example of photochemical production of cyclic- $\mathrm{N}_{3}$ and more importantly one

${ }^{a}$ State Key Laboratory for Molecular Dynamics, Dalian Institute of Chemical Physics, 457 Zhongshan Rd. Dalian, Liaoning, 116023, P. R. China

${ }^{b}$ Department of Physics, Dalian University of Technology, Dalian, China

${ }^{c}$ Cherry L. Emerson Center for Scientific Computation and Department of Chemistry, Emory University, Atlanta, Georgia, 30322, USA

${ }^{d}$ Department of Chemistry and Biochemistry, University of California, Santa Barbara, CA 93106-9510, USA.

E-mail:wodtke@chem.ucsb.edu where high-level quantum chemical methods could be used to guide our analysis of the microscopic reaction mechanism, we undertook a study of the photochemistry of hydrazoic acid, $\mathrm{HN}_{3}$, the simplest member of the $\mathrm{XN}_{3}$ family.

The UV photochemistry of $\mathrm{HN}_{3}$ (especially in its first excited singlet state, $S_{1}$, possessing $A^{\prime \prime}$ symmetry) has attracted much attention; one might even refer to it as a benchmark system for the study of polyatomic ultraviolet photochemical dynamics. All four of the products of $\mathrm{HN}_{3}$ photolysis:

$$
\begin{aligned}
\mathrm{HN}_{3} & \rightarrow \mathrm{H}+\mathrm{N}_{3} \Delta E=88.7 \mathrm{kcal} \mathrm{mol}^{-1} \text { - Channel } \\
& \rightarrow \mathrm{NH}\left(\mathrm{a}^{1} \Delta\right)+\mathrm{N}_{2} \Delta E=63.5 \mathrm{kcal} \mathrm{mol}^{-1} \text { - Channel }
\end{aligned}
$$

are suitable for efficient detection by optical means, thus the reaction has been studied with several quantum-state resolved methods. ${ }^{19-37} \mathrm{HN}_{3}$ is also particularly amenable to high level $a b$ initio quantum chemistry methods in both ground and excited electronic states, ${ }^{38-40}$ making comparison between experiment and theory particularly useful for the analysis of its photochemistry.

It is noteworthy that the possible formation of cyclic- $\mathrm{N}_{3}$ in $\mathrm{HN}_{3}$ photochemistry has never been the focus of any of these many high quality studies. Channel (2), which dominates at photolysis wavelengths where branching ratios have been measured, ${ }^{29}$ has attracted much of the attention. ${ }^{19-26}$ Of those experiments focused on channel (1), ${ }^{27-32}$ most employed photolysis wavelengths where cyclic- $\mathrm{N}_{3}$ was not energetically accessible, $\lambda>244 \mathrm{~nm}$, or employed methods that would not reveal the production of this molecule.

The H-atom Rydberg time-of-flight (HRTOF) ${ }^{41}$ method is one of the few possible means by which the formation of cyclic- $\mathrm{N}_{3}$ in $\mathrm{HN}_{3}$ photolysis might be observed and studied. Furthermore, it has already proven useful in understanding the dynamics of channel (1). ${ }^{27,28}$ Cook et al. reported HRTOF 
results for photolysis wavelengths between 240 and $280 \mathrm{~nm}$, providing comprehensive characterization of the energy release to photoproducts after excitation to the $S_{1}$ state. ${ }^{28}$ Also demonstrating the power of comparison between experiment and theory for this system, analysis with an ab initio potential energy surface using wave packet calculations helped support the vibrational assignment of the well resolved features in the HRTOF energy distribution. ${ }^{39}$ This work produced no evidence of cyclic- $\mathrm{N}_{3}$ formation despite the fact that some of the reported photolysis wavelengths surpassed its energetic formation threshold. ${ }^{42}$ Zhang et al. are the only group to report HRTOF results on $\mathrm{HN}_{3}$ at shorter wavelengths still; they carried out photolysis experiments at 248 and $193 \mathrm{~nm} .{ }^{27}$ While these results do not reveal the full nature of the wavelength dependence of channel (1), photolysis at 193-nm clearly leads to more internally excited $\mathrm{N}_{3}$ molecules than does photolysis at all other wavelengths that have been studied. ${ }^{27}$ This suggested to us the value of a more comprehensive and systematic study of the wavelength dependent dynamics of photochemical H-production in $\mathrm{HN}_{3}$.

Recently, we have completed such a study using the HRTOF method, spanning the range of photolysis wavelengths between 280 and $188 \mathrm{~nm}$ in roughly $5 \mathrm{~nm}$ steps. A full analysis of these results will be reported later. This paper focuses on the 5-6 eV energy range (225-199 nm), where a marked transition in the photochemical dynamics is observed. We observe many of the same features seen previously corresponding to formation of linear azide, where on average $40 \%$ of the available energy is channeled to translation. In addition, these results reveal a low-translational-energy, H-atom producing channel that grows in importance above a threshold of $\sim 5.6 \mathrm{eV}$. The translational energy release of this "slow component" is consistent with known thermodynamics for formation of cyclic- $\mathrm{N}_{3}$ Furthermore, a comparison to high level $a b$ initio quantum chemistry calculations reveals that the experimentally observed threshold for this channel is coincident with an $\mathrm{N}_{3}$-ring closing transition state for $\mathrm{HN}_{3}$ on its $\mathrm{S}_{1}$ potential energy surface that leads to an electronically excited cyclic intermediate of $\mathrm{HN}_{3}$. Theory shows that this intermediate may dissociate over a small barrier to $\mathrm{H}+$ cyclic- $\mathrm{N}_{3}$. These results provide the first insight into the microscopic mechanism for $\mathrm{N}_{3}$ ring closure in $\mathrm{XN}_{3}$ photochemistry.

\section{Methodology}

We used a multidisciplinary methodology to approach this problem combining high level HRTOF experiments with the best available quantum chemistry methods.

\section{Experiment}

The H/D-Rydberg-atom-time-of-flight (HRTOF) technique has been described in detail elsewhere ${ }^{43,44}$ and only a brief description of the more salient points is presented here. A skimmed, pulsed, molecular beam of $\mathrm{HN}_{3}$, seeded in helium (mixing ratio $\sim 2 \%$, total pressure $\sim 1 \mathrm{~atm}$ ), is crossed at $90^{\circ}$ with the output of the photolysis laser (UV power $0.1-4 \mathrm{~mJ}$, with the beam diameter $\geq 0.1 \mathrm{~cm}$ ), which consists of $\mathrm{Nd}$ :YAG (Spectra Physics Pro-290) 3rd harmonic pumped dye laser (Sirah, PESC-G-24) system. The second harmonic of the dye laser light is generated using a BBO crystal (tunable in the range of $225-280 \mathrm{~nm}$ ). Photons in the range of 188 to $225 \mathrm{~nm}$ are generated by combining the output of the second harmonic of the dye laser with the Nd:YAG fundamental $(1064 \mathrm{~nm})$, using a second BBO crystal. Distributions of recoiling H/D atoms are probed using a detector positioned at different lab angles. After a short time delay $(\sim 10 \mathrm{~ns})$ recoiling $\mathrm{H}$-atom products from the photodissociation were tagged by excitation to high-n Rydberg levels using a two color scheme: Lyman- $\alpha$ radiation at $121.6 \mathrm{~nm}$ followed by UV radiation at $\sim 366 \mathrm{~nm}$. Photons at the Lyman- $\alpha$ wavelength are generated by fourwave mixing of two $212.5 \mathrm{~nm}$ photons and one $845 \mathrm{~nm}$ photon in a cell filled with a $3: 1$ ratio $\mathrm{Ar}-\mathrm{Kr}$ mixture of at a total pressure about 70 Torr. Photons at $212.5 \mathrm{~nm}$ are produced by doubling the output of a Nd:YAG (Spectra Physics Pro-290) 3rd harmonic pumped dye laser (Sirah, PESC-G-24) operating at $\sim 425 \mathrm{~nm}$. A portion of the $532 \mathrm{~nm}$ output of the YAG is used to pump another dye laser (Continuum ND6000) operating at $\sim 845 \mathrm{~nm}$. These beams are then focused into a cell with $\mathrm{Kr} / \mathrm{Ar}$ mixing gas where four wave mixing at $121.6 \mathrm{~nm}$ is generated. The remainder of the $532 \mathrm{~nm}$ source is used to pump a third Radiant dye laser (Radiant Dye Lasers- Jaguar, D90MA), operating at $\sim 732 \mathrm{~nm}$, the output from which frequency is doubled to $\sim 366 \mathrm{~nm}$, and used to promote the H-atoms from the $n=2$ level to a Rydberg state of high principal quantum number $(n=30-90)$, lying just below the ionization threshold. The beam paths of both tagging lasers have been set to ensure their maximum temporal overlap in the interaction region, and all three beams are focused so as to ensure their maximum mutual spatial overlap within this volume. Any charged species formed at the volume of tagging by initial laser excitation are extracted away from the TOF axis by a small electric field placed across the interaction region.

The tagged $\mathrm{H}$-atoms then fly away from the interaction region to reach a micro-channel plate (MCP) detector. The Rydberg $\mathrm{H}$-atoms are efficiently ionized by the strong electric field applied to the MCP detector. The total distance from the interaction region to the front face of the detector is $\sim 333$ $\mathrm{mm}$. The signal obtained is amplified by a preamplifier, discriminated by a discriminator, sent to both a digital oscilloscope for visual display and a multi-channel scaler (P7888-2(E) FASTCOMTEC) for accumulation (typically more than $10^{5}$ laser shots per TOF profile) and subsequent data analysis.

$\mathrm{HN}_{3}$ was prepared by heating sodium azide $\left(\mathrm{NaN}_{3}\right)$ in excess stearic acid under vacuum for $3-4 \mathrm{~h}$ at $\sim 80-100{ }^{\circ} \mathrm{C}$. $\mathrm{DN}_{3}$ was produced by reacting $\mathrm{NaN}_{3}$ with an excess of deuterated phosphoric acid, which is generated by reacting $\mathrm{D}_{2} \mathrm{O}$ with $\mathrm{P}_{2} \mathrm{O}_{5}$ under vacuum conditions. The $\mathrm{H}(\mathrm{D}) \mathrm{N}_{3}$ sample was stored in a stainless steel container and $\mathrm{He}$ gas was filled to produce $1-2 \% \mathrm{H}(\mathrm{D}) \mathrm{N}_{3} / \mathrm{He}$ ratio (the total pressure made up to $\sim 1-4$ atm with pure helium). Purity was checked by mass spectrometry (SRS, RGA200).

\section{Theory}

Geometries of potential energy minima and transition states were optimized using the analytical gradient at the statespecific (ss)-CASSCF (complete active space self-consistent 
field) level. ${ }^{45}$ In ss-CASSCF calculations, the full valence active space, (16e/13o), consisting of 16 electrons in 13 molecular orbitals, was used with the cc-pVTZ (VTZ), Dunning's correlation consistent polarized valence triple-zeta basis set. ${ }^{46}$ The Hessian matrix, vibrational frequencies and the zero point energy (ZPE) were calculated by numerical differentiation of the energy gradient at the same ss-CASSCF/VTZ level as in geometry optimization. Energetics were further refined by the internally contracted configuration interaction with single and double excitations (MRCISD) ${ }^{47,48}$ plus multi-reference version of Davidson's correction $(\mathrm{Q})^{49,50}$ with the Dunning's correlation consistent augmented polarized valence triple zeta basis (AVTZ), ${ }^{46}$ where a pre-determined ss-CASSCF (16e/ 13o)/AVTZ wave function was selected as the reference configuration. In the MRCISD calculation, only the 1s orbitals of nitrogen atoms were kept doubly occupied in all configurations and the remaining 16 electrons were correlated, denoted as MRCISD(Q)(16e/13o)/AVTZ. MOLPRO 2002.6 $6^{51}$ was used for all the CASSCF and MRCISD calculations.

\section{Results and discussion}

Fig. 1 shows the center-of-mass translational energy release distribution for the $\mathrm{H}(\mathrm{D})+\mathrm{N}_{3}$ channel at a few selected photolysis wavelengths obtained by direct inversion of the raw TOF data obtained at the magic recoil angle $\left(\Theta=54.7^{\circ}\right)$ with respect to the original direction of the polarization of the photolysis laser. This angle is useful in that signal intensities are thereby independent of the $\mathrm{H}$-atom angular distribution. In each panel, the isotope (H or D) is indicated as is the photolysis wavelength in $\mathrm{nm}$. For example, D-208 was a D-atom Rydberg tagging experiment at a photolysis wavelength of $208 \mathrm{~nm}$. The downward pointing arrows indicate the thermodynamically-determined limit to the translational
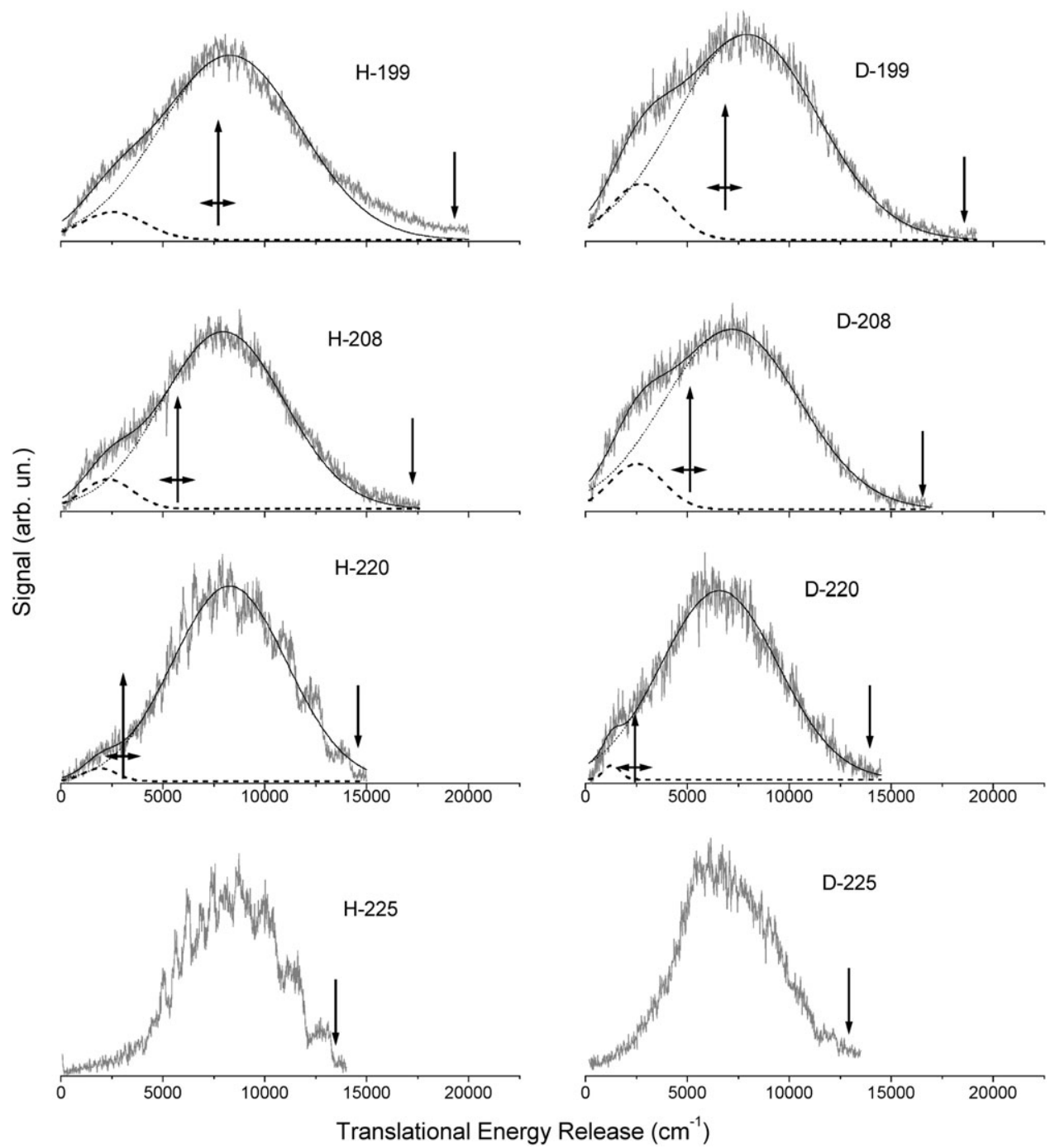

Fig. 1 H-Atom Rydberg TOF derived translational energy release distributions for $\mathrm{HN}_{3}$ and $\mathrm{DN}_{3}$ photolysis at representative wavelengths. Two channels are observed at wavelengths shorter than about $220 \mathrm{~nm}$. The low translational energy channel (dashed line) is consistent in energy release with formation of cyclic- $\mathrm{N}_{3}$, the formation of which cannot lead to translational energies larger than that shown by the upward directed arrows. The high translational energy release channel (dotted line) produces linear- $\mathrm{N}_{3}$ which has a thermodynamically defined limit to the translational energy release shown by the downward pointing arrows. The double headed arrows represent the uncertainty in our knowledge of the heat of formation of cyclic- $\mathrm{N}_{3}$. The notation on each panel indicates the isotope (D or H) as well as the photolysis wavelength in $\mathrm{nm}$. 
energy release assuming the detected H(D)-atoms are produced with linear $\mathrm{N}_{3}$-radicals. The partially resolved structure seen in panel H-225 can also be successfully assigned to vibrational states of the linear $\mathrm{N}_{3}$-radical as in ref. 27 and 28.

At photolysis wavelengths less than or equal to $\sim 220 \mathrm{~nm}$, a second component to the translational energy release is clearly observable, and is notably stronger for $\mathrm{D}$ than for $\mathrm{H}$. This component exhibits a smaller translational energy release, suggesting formation of a form of $\mathrm{N}_{3}$ with high internal energy. Energy release distributions corresponding to photolysis wavelengths longer than $225 \mathrm{~nm}$ qualitatively resemble the $225 \mathrm{~nm}$ results and show no sign of this second lowtranslational-energy component. In order to better quantify the two contributions to the translational energy release distribution, the data was fit to two Gaussian functions, shown as dashed and dotted lines in Fig. 1. One can see that the data is well fit by this simple strategy. The upward pointing arrows (best value) and double-headed horizontal arrow (error bars) show the thermodynamically-determined limit of translational energy release assuming formation of $\mathrm{H}(\mathrm{D})$ with cyclic- $\mathrm{N}_{3} \cdot{ }^{15,16}$

The fraction of the $\mathrm{H}(\mathrm{D})$-producing photochemistry that results in the high energy form of $\mathrm{N}_{3}$ can be estimated by integrating the Gaussian components used to fit the translational energy release distributions of Fig. 1. This is shown for both isotopes in Fig. 2 for photolysis wavelengths: 220, 217 , 213, 208, 203 and $199 \mathrm{~nm}$. We found no detectible branching between channels at longer photolysis wavelengths. The observed threshold at a photolysis energy of $5.65 \pm 0.05 \mathrm{eV}$

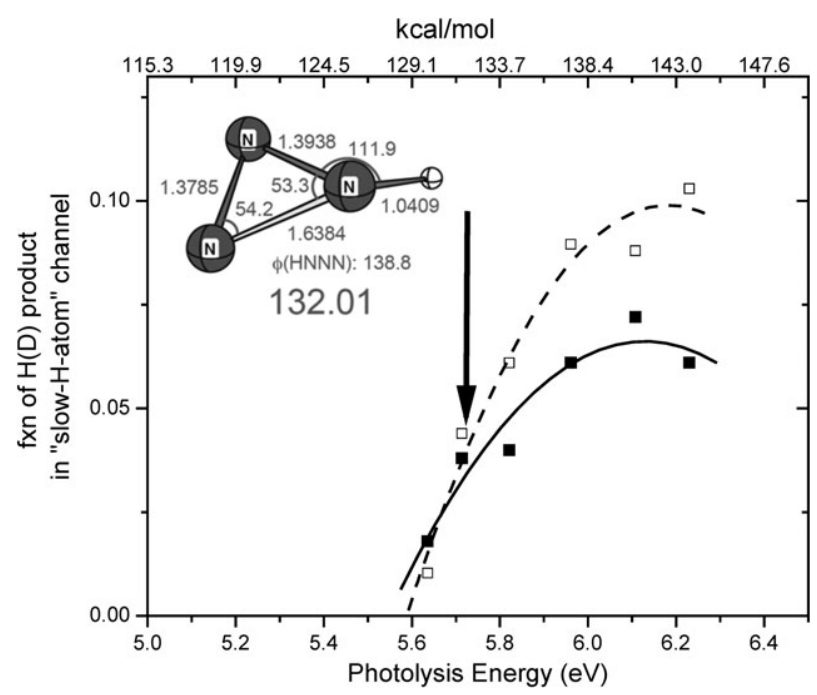

Fig. 2 Observations of barriers in the $S_{1}$ state of $H_{3}$. The solid squares show the derived fraction of $\mathrm{H}$-atom channel appearing at low translational energies. This represents the fraction of $\mathrm{H}$-atoms formed together with a high energy form of $\mathrm{N}_{3}$ (possibly cyclic- $\mathrm{N}_{3}$ ). The solid arrow shows the theoretically predicted threshold energy for ringclosure on the $\mathrm{S}_{1}$ surface of $\mathrm{HN}_{3}$. The accuracy of this prediction is expected to be better than $\pm 0.2 \mathrm{eV}$. The structure of the calculated transition state to ring closure on the $\mathrm{S}_{1}$ surface is shown as an inset. This comparison between theory and experiment provides strong evidence for the observation of ring closure in the UV photolysis of $\mathrm{HN}_{3}$. Open squares show the results of $\mathrm{DN}_{3}$ photolysis. The isotope effect favoring D-atoms may be indicative of competition between direct dissociation and ring-closure. compares favorably with the calculated barrier to ring closure on the $\mathrm{S}_{1}$ surface at $5.72 \mathrm{eV}$ (shown as the solid arrow). The structure of this transition state is shown as an inset to Fig. 2. Fig. 2 also reveals a marked "inverse" isotope effect increasing with incidence energy, where $\mathrm{DN}_{3}$ leads with about twice the efficiency as $\mathrm{HN}_{3}$ to the high energy form of $\mathrm{N}_{3}$, reaching a maximum of $\sim 10 \%$ of the radical channel at about $6 \mathrm{eV}$. Note that the observed threshold is well below the energetic threshold for spin allowed secondary dissociation to form $\mathrm{H}+\mathrm{N}_{2}+$ $\mathrm{N}\left({ }^{2} \mathrm{D}\right)$, which requires $6.2 \mathrm{eV}$.

Fig. 3 shows the energetics of the relevant pathway for the formation of the cyclic- $\mathrm{N}_{3}$ species on the $\mathrm{S}_{1}$ potential surface, extracted from an extensive and highly accurate quantum chemical study of the $S_{0}, S_{1}, T_{1}$ and $S_{2}$ potential energy surfaces. Here, energies (with zero point energy correction) are shown in $\mathrm{kcal} \mathrm{mol}^{-1}$ along with the optimized structures for local minima and saddle points. Both the barriers to ring closure and to dissociation of the ring closed intermediate are in good agreement with the experimentally observed threshold for the low-translational-energy $\mathrm{H}$-atom producing channel. This leads us to postulate a reasonable mechanism for cyclic$\mathrm{N}_{3}$ formation in $\mathrm{HN}_{3}$ photolysis (Fig. 3); namely, the excitation of $\mathrm{HN}_{3}$ in this energy range leads to the ring closing transition state with observable efficiency and subsequent formation of the ring-closed intermediate that may then dissociate to the products.

The threshold of the low-translational-energy channel at $5.65 \mathrm{eV}$ is close to the onset of a strong $\mathrm{HN}_{3}$ absorption that peaks near $210 \mathrm{~nm},{ }^{52}$ which we attribute to the $\mathrm{S}_{0}\left(\mathrm{~A}^{\prime}\right) \rightarrow$ $\mathrm{S}_{2}\left(\mathrm{~A}^{\prime}\right)$ absorption. The slow channel production efficiency also shows a leveling off at $\sim 6.1 \mathrm{eV}$ mimicking the energy dependence of the $\mathrm{S}_{0} \rightarrow \mathrm{S}_{2}$ absorption spectrum. In addition, we observe a rapidly changing value of $\beta$ near the threshold indicative of the increasing importance of the $S_{0} \rightarrow S_{2}$ absorption. Specifically $\beta$ 's are between -1 and -0.5 for the "fast channel", resulting from $\mathrm{S}_{0}\left(\mathrm{~A}^{\prime}\right) \rightarrow \mathrm{S}_{1}\left(\mathrm{~A}^{\prime \prime}\right)$, but near 0 for the "slow channel". It is therefore reasonable to suspect the $\mathrm{S}_{2}$-state and its subsequent photodynamics as playing an important role in the production of low translational energy $\mathrm{N}_{3}+\mathrm{H}$. Our ab-initio calculations on the $\mathrm{S}_{2}$-state show a trans-minimum with the structure shown in Table 1 and a seam of crossing (conical intersection) between the $\mathrm{S}_{2}$ - and the $\mathrm{S}_{1}$-state near the trans-minimum structure of the $\mathrm{S}_{2}$ state, also shown in Table 1. Considering the structure of the ground state (also shown in Table 1) excitation to the $\mathrm{S}_{2}$ Franck-Condon region would result in $\mathrm{N}-\mathrm{N}-\mathrm{N}$ bending and $\mathrm{N}-\mathrm{N}$ stretching between both $\mathrm{N}-\mathrm{N}$-bonds as well as some HNN bending and stretching motion. Molecules excited to $\mathrm{S}_{2}$ and subsequently transmitted to the $S_{1}$-state through the $S_{2}-S_{1}$ seam crossing (conical intersection) would be excited exactly with the proper kind of vibration to provide access to the ring closed transition state of $S_{1}$. Thus, the internal conversion to $\mathrm{S}_{1}$ state will be a dominant process with low excitation energy. Thus excitation to $S_{2}$ followed by $S_{2} \rightarrow S_{1}$ internal conversion appears to offer a reasonable explanation for the mechanism of ring closure.

Our ab initio calculations also revealed a nearby conical intersection that could transmit $S_{2}$ population to $S_{0}$. An alternative mechanism for slow atom formation was therefore 

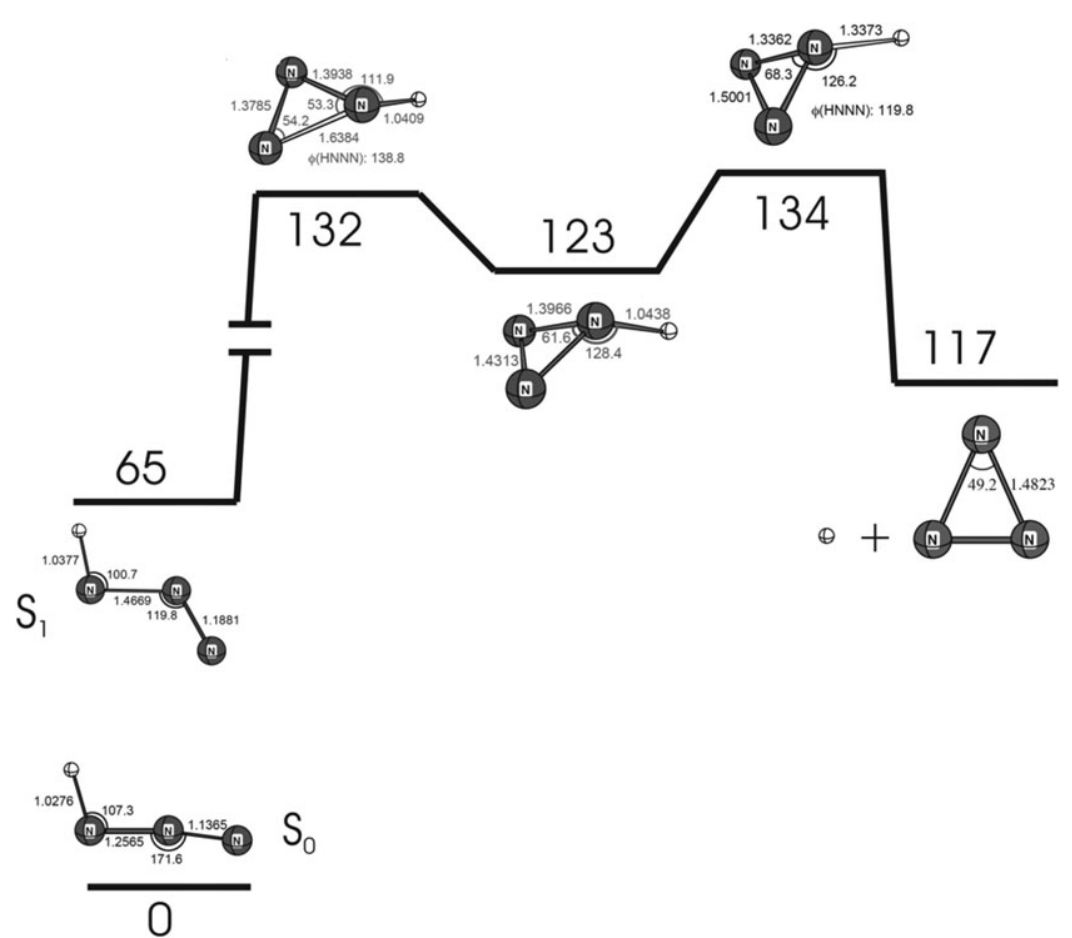

Fig. 3 Postulated cyclization mechanism in $\mathrm{HN}_{3}$ photolysis. Ab initio quantum chemical calculations of isomerization on the $\mathrm{S}_{1}$ state of $\mathrm{HN}_{3}$. All energies are shown in kcal mol${ }^{-1}$ and are corrected for zero-point energy. The near linear $\mathrm{N}_{3}$ in the $\mathrm{S}_{0} \mathrm{HN}_{3}$ is thought to be strongly excited in the bend and $\mathrm{N}-\mathrm{N}$ stretch leading some reactive flux to the ring-closing barrier and formation of the electronically excited cyclized $\mathrm{HN}_{3}$. This intermediate may dissociate producing cyclic- $\mathrm{N}_{3}$.

considered. Here we modeled the fast $\mathrm{H}$-atom production channel as derived from $\mathrm{S}_{0}-\mathrm{S}_{1}$ excitation and slow atom production as resulting from $\mathrm{S}_{0}-\mathrm{S}_{2}$ excitation followed by $\mathrm{S}_{2}-\mathrm{S}_{0}$ internal conversion and statistical decomposition on the $\mathrm{S}_{0}$ state. We used an RRKM program ${ }^{53}$ to model the branching between reactions (1) and (2) on $\mathrm{S}_{0}$ for both isotopomers, employing reactant and transition state frequencies and energies obtained from our electronic structure calculations. See Table 2. Two major discrepancies with observation arise from this analysis. First, the RRKM analysis predicts a significant (1.7) normal, i.e. favoring $\mathrm{H}$-atom production, isotope effect; whereas experiment reveals an isotope effect of similar magnitude but opposite direction, i.e. favoring D-atom production. Furthermore, the RRKM predictions cannot reproduce the energy dependence of the isotope effect apparent in Fig. 2. Second, due to the fact that the $\mathrm{S}_{2}$ absorption is growing rapidly with energy $(\sim 100 \times$ stronger than the $\mathrm{S}_{1}$ absorption at $6 \mathrm{eV}$ ), this model predicts that about $90 \%$ of all $\mathrm{H}$-atoms would appear in the slow channel (at $6 \mathrm{eV}$ ); whereas experiment shows only $5-10 \%$. This analysis strongly suggests that $\mathrm{S}_{2}-\mathrm{S}_{0}$ internal conversion does not play a role in the production of slow atoms.

In addition to the $S_{1}$ ring-closing transition state, the transition state for 1,2 $\mathrm{H}$-atom migration, producing a planar minimum with $\mathrm{H}$ attached to the middle $\mathrm{N}$ atom, was also found. This barrier is, however, $4.5 \mathrm{kcal} \mathrm{mol}^{-1}$ higher in energy than the ring closing barrier. More importantly, in going from the $\mathrm{S}_{0}$ structure, access to the $1,2 \mathrm{H}$-atom migration transition state requires a large HNN bending motion, while the ring closure transition state is associated with a large NNN bending motion. The geometrical differences between the $\mathrm{S}_{2}$ and/or $\mathrm{S}_{1}$ state global minimum and the

Table 1 Theoretical structure (in $\AA$ and ${ }^{\circ}$ ) of the $S_{2}$ state and the energy-minimum of the seam crossing (MSX, conical intersection) from $\mathrm{S}_{2}$ to $\mathrm{S}_{1}$. The structure of the ground state minimum $\left(\mathrm{S}_{0}\right)$ is shown for comparison. All the structures except $\mathrm{S}_{1}$ ring-closing TS are coplanar $\left(\phi_{\mathrm{H}-\mathrm{N} 1-\mathrm{N} 2-\mathrm{N} 3}\right.$ $\left.=180.0^{\circ}\right)^{a}$. Notice how $\mathrm{S}_{0} \rightarrow \mathrm{S}_{2} \rightarrow \mathrm{S}_{1}$ leads toward the structure of the $\mathrm{S}_{1}$ ring closing TS. This is particularly evident for the $\mathrm{N}_{1}-\mathrm{N}_{3}$ distance and the $\mathrm{N}_{1}-\mathrm{N}_{2}-\mathrm{N}_{3}$ angle, which are critical to cyclization

\begin{tabular}{lllllrr}
\hline & $R_{\mathrm{H} 1-\mathrm{N} 1}$ & $R_{\mathrm{N} 1-\mathrm{N} 2}$ & $R_{\mathrm{N} 2-\mathrm{N} 3}$ & $\Theta_{\mathrm{H}-\mathrm{N} 1-\mathrm{N} 2}$ & $\Theta_{\mathrm{N} 1-\mathrm{N} 2-\mathrm{N} 3}$ & $R_{\mathrm{N} 1-\mathrm{N} 3}$ \\
\hline $\mathrm{S}_{\mathrm{O}}$-min & $1.028(1.001)$ & $1.257(1.251)$ & $1.136(1.143)$ & $107.3(108.8)$ & $171.6(171.4)$ & $2.386(2.388)$ \\
$\mathrm{S}_{2}$-trans-min & $(0.992)$ & $(1.301)$ & $(1.286)$ & $(134.9)$ & $(102.2)$ & $(2.013)$ \\
$\mathrm{S}_{2}-\mathrm{S}_{1}$ MSX & $(0.992)$ & $(1.302)$ & $(1.287)$ & $(136.7)$ & $(102.1)$ & $(2.014)$ \\
$\mathrm{S}_{1}$-ring closing TS & 1.041 & 1.393 & 1.378 & 1.50 .7 & 1.638 & 119.8 \\
$\mathrm{~S}_{1}$-trans-min & 1.038 & 1.467 & 1.188 & 100.7 & 2.301
\end{tabular}

${ }^{a}$ The geometric parameters in parentheses were optimized at 3 states $\left(\mathrm{S}_{0}, \mathrm{~S}_{1}\right.$ and $\mathrm{S}_{2}$ with equal weight) averaged CASSCF(16e/12o)/cc-pVTZ level of theory with $\sigma^{*}{ }_{\mathrm{N}-\mathrm{H}}$ orbital removed from the full valence CASSCF space. 
Table 2 Input data for RRKM calculations ${ }^{a}$

\begin{tabular}{lrlllllr}
\hline & \multicolumn{1}{c}{$E_{0}$} & $\nu_{1}$ & $\nu_{2}$ & \multicolumn{1}{c}{$\nu_{3}$} & \multicolumn{1}{c}{$\nu_{4}$} & \multicolumn{1}{c}{$\nu_{5}$} & \multicolumn{1}{c}{$\nu_{6}$} \\
\hline $\mathrm{HN}_{3}$ & 0.0 & 3386 & 2189 & 1313 & 1155 & 601 & 524 \\
$\mathrm{H}+\mathrm{N}_{3} \mathrm{TS}^{b}$ & 86.5 & ${ }^{c}$ & 1645 & 1320 & 595 & 505 & 95 \\
$\mathrm{NH}+\mathrm{N}_{2} \mathrm{TS}$ & 50.5 & 3233 & 2340 & $-^{c}$ & 739 & 157 & 120 \\
$\mathrm{DN}$ & 0.0 & 2475 & 2181 & 1172 & 1003 & 589 & 492 \\
$\mathrm{D}+\mathrm{N}_{3} \mathrm{TS}^{b}$ & 87.8 & $\bar{c}^{c}$ & 1645 & 1320 & 595 & 505 & 95 \\
$\mathrm{ND}+\mathrm{N}_{2}$ TS & 50.7 & 2362 & 2340 & $-^{c}$ & 540 & 124 & 116
\end{tabular}

${ }^{a} E_{0}$ (corrected for zero point energy) in $\mathrm{kcal} \mathrm{mol}^{-1}$, vibrational frequencies in $\mathrm{cm}^{-1} \cdot{ }^{b}$ No barrier to dissociation was found. Transition state for $\mathrm{N}-\mathrm{H}(\mathrm{D})$ bond cleavage was taken as the products $\mathrm{H}(\mathrm{D})+\mathrm{N}_{3} \cdot{ }^{c}$ Imaginary or zero.

Franck-Condon geometry suggests that the ring-closure transition state is more easily accessible than the 1,2 $\mathrm{H}$-migration transition state and is more likely to be important.

A word regarding the "inverse" isotope effect is also in order. An unambiguous interpretation awaits detailed theoretical analysis; however, we speculate that the time available for isomerization to the cyclic structure is approximately double for $\mathrm{DN}_{3} v s . \mathrm{HN}_{3}$. Remember that isomerization to the cyclic intermediate proceeds in competition with $\mathrm{H}(\mathrm{D})$-atom elimination to form linear $\mathrm{N}_{3}$. The large negative anisotropy parameter $(\beta \sim-0.7)^{29}$ for this process indicates sub-picosecond dissociation dynamics. As velocities of the D-atom undergoing direct dissociation will be approximately half that of $\mathrm{H}$-atoms, it is possible that more time is available for the $\mathrm{N}$-atom motion required to close the ring in the case of the heavy departing atom.

In summary, a wavelength dependent study using $\mathrm{H}$-atom Rydberg time-of-flight revealed an energetic threshold for a previously unobserved dissociation pathway in the UV photolysis of hydrazoic acid, exhibiting a threshold that is coincident with a calculated barrier to ring closing on the $\mathrm{S}_{1}\left(\mathrm{~A}^{\prime \prime}\right)$ potential energy surface. Theory shows that passage over this barrier leads to a local minimum in the potential, which exhibits an $\mathrm{N}_{3}$-ring-closed, non-planar structure, which may dissociate to cyclic- $\mathrm{N}_{3}+\mathrm{H}$. The translational energy release of the newly observed dissociation channel is consistent with the thermodynamics of cyclic- $\mathrm{N}_{3}$ formation. Through analysis of all experimental observations we propose a detailed photophysical mechanism for cyclic- $\mathrm{N}_{3}$ formation in $\mathrm{HN}_{3}$ photolysis. Specifically, we propose that $S_{0} \rightarrow S_{2}$ photo-excitation is followed by $S_{2} \rightarrow S_{1}$ internal conversion through a conical intersection located near the energy minimum of the $S_{2}$ state and traversal of the cyclization barrier on $\mathrm{S}_{1}$ and subsequent $\mathrm{N}-\mathrm{H}$ bond cleavage. We have analyzed other possible mechanisms of producing "slow atoms" and found them inconsistent with experimental observations.

\section{Acknowledgements}

AMW acknowledges support from an AFOSR grant No. FA9550-04-1-0057. KM acknowledges support from AFOSR grants No. FA9550-04-1-0080 and FA9550-04-1-0321, as well as a computer-time grant under the DoD High Performance Computing Program.

\section{References}

1 L. J. Wang and P. G. Mezey, J. Phys. Chem. A, 2005, 109, 3241-43.

2 D. L. Strout, J. Phys. Chem. A, 2004, 108, 10911-16.

3 L. Y. Bruney, T. M. Bledson and D. L. Strout, Inorg. Chem., 2003, 42, 8117-20.

4 K. J. Wilson, S. A. Perera, R. J. Bartlett and J. D. Watts, J. Phys. Chem. A, 2001, 105, 7693-99.

5 M. Tobita and R. J. Bartlett, J. Phys. Chem. A, 2001, 105, 4107-13.

6 M. N. Glukhovtsev, H. J. Jiao and P. V. Schleyer, Inorg. Chem., 1996, 35, 7124-33.

7 A. Vij, J. G. Pavlovich, W. W. Wilson, V. Vij and K. O. Christe, Angew. Chem., Int. Ed., 2002, 41, 3051-54.

8 K. O. Christe, W. W. Wilson, J. A. Sheehy and J. A. Boatz, Angew. Chem., Int. Ed., 1999, 38, 2004-09.

9 N. Hansen and A. M. Wodtke, J. Phys. Chem. A, 2003, 107, 10608-14.

10 P. C. Samartzis, J. J. Lin, T. T. Ching, C. Chaudhuri, Y. T. Lee, S. H. Lee and A. M. Wodtke, J. Chem. Phys., 2005, 123, 051101-1-4.

11 N. Hansen, A. M. Wodtke, S. J. Goncher, J. Robinson, N. Sveum and D. M. Neumark, J. Chem. Phys., 2005, 123, 104305.

12 A. M. Wodtke, N. Hansen, J. C. Robinson, N. E. Sveum, S. J. Goncher and D. M. Neumark, Chem. Phys. Lett., 2004, 391, 334-37.

13 N. Hansen, A. M. Wodtke, A. V. Komissarov, K. Morokuma and M. C. Heaven, J. Chem. Phys., 2003, 118, 10485-93.

14 N. Hansen, A. M. Wodtke, A. V. Komissarov and M. C. Heaven, Chem. Phys. Lett., 2003, 368, 568-73.

15 M. Bittererova, H. Ostmark and T. Brinck, J. Chem. Phys., 2002, 116, 9740-48.

16 P. Zhang, K. Morokuma and A. M. Wodtke, J. Chem. Phys., 2005, 122, 014106-1-11.

17 D. Babikov, B. Kendrick, P. Zhang and K. Morokuma, J. Chem. Phys., 2005, 122, 044315.

18 D. Babikov, P. Zhang and K. Morokuma, J. Chem. Phys., 2004, 121, 6743-49.

19 R. J. Barnes, A. Gross, M. Lock and A. Sinha, J. Phys. Chem. A, 1997, 101, 6133-37.

20 M. H. Alexander, H. J. Werner and P. J. Dagdigian, J. Chem. Phys., 1988, 89, 1388-400.

21 G. Schonnenbeck, H. Biehl, F. Stuhl, U. Meier and V. Staemmler, J. Chem. Phys., 1998, 109, 2210-19.

22 K. H. Gericke, M. Lock, R. Fasold and F. J. Comes, J. Chem. Phys., 1992, 96, 422-32.

23 K. H. Gericke, T. Haas, M. Lock, R. Theinl and F. J. Comes, J. Phys. Chem., 1991, 95, 6104-11.

24 H. H. Nelson and J. R. McDonald, J. Chem. Phys., 1990, 93, 8777-83.

25 F. Rohrer and F. Stuhl, J. Chem. Phys., 1988, 88, 4788-99.

26 J. J. Chu, P. Marcus and P. J. Dagdigian, J. Chem. Phys., 1990, 93, 257-67.

27 J. S. Zhang, K. S. Xu and G. Amaral, Chem. Phys. Lett., 1999, 299, 285-90.

28 P. A. Cook, S. R. Langford and M. N. R. Ashfold, Phys. Chem. Chem. Phys., 1999, 1, 45-55.

29 M. Lock, K. H. Gericke and F. J. Comes, Chem. Phys., 1996, 213, 385-96.

30 T. Haas, K. H. Gericke, C. Maul and F. J. Comes, Chem. Phys. Lett., 1993, 202, 108-14.

31 K. H. Gericke, M. Lock and F. J. Comes, Chem. Phys. Lett., 1991, 186, 427-30.

32 K. H. Gericke, R. Theinl and F. J. Comes, Chem. Phys. Lett., 1989, 164, 605-11.

33 R. Pahnke, S. H. Ashworth and J. M. Brown, Chem. Phys. Lett., 1988, 147, 179-82.

34 M. Hawley, A. P. Baronavski and H. H. Nelson, J. Chem. Phys., 1993, 99, 2638-42.

35 R. A. Beaman, T. Nelson, D. S. Richards and D. W. Setser, J. Phys. Chem., 1987, 91, 6090-92.

36 C. R. Brazier, P. F. Bernath, J. B. Burkholder and C. J. Howard, J. Chem. Phys., 1988, 89, 1762-67.

37 G. Chambaud and P. Rosmus, J. Chem. Phys., 1992, 96, 77-89.

38 U. Meier and V. Staemmler, J. Phys. Chem., 1991, 95, 6111-17. 
39 P. A. Cook, P. Jimeno, M. N. R. Ashfold, G. G. Balint-Kurti and R. N. Dixon, Phys. Chem. Chem. Phys., 2002, 4, 1513-21.

40 W. H. Fang, J. Phys. Chem. A, 2000, 104, 4045-50.

41 L. Schnieder, K. Seekamprahn, J. Borkowski, E. Wrede, K. H. Welge, F. J. Aoiz, L. Banares, M. J. Dmello, V. J. Herrero, V. S. Rabanos and R. E. Wyatt, Science, 1995, 269, 207-10.

42 We will show that this result is consistent with the present results as $\mathrm{HN}_{3}$ photolysis cannot produce cyclic $\mathrm{N}_{3}$ until the energy is well above the threshold.

43 L. Schnieder, K. SeekampRahn, E. Wrede and K. H. Welge, J. Chem. Phys., 1997, 107, 6175-95.

44 L. Schnieder, W. Meier, K. H. Welge, M. N. R. Ashfold and C. M. Western, J. Chem. Phys., 1990, 92, 7027-37.

45 H. J. Werner and P. J. Knowles, J. Chem. Phys., 1985, 82, 5053-63.

46 T. H. Dunning, J. Chem. Phys., 1989, 90, 1007-23.

47 H. J. Werner and P. J. Knowles, J. Chem. Phys., 1988, 89, 5803-14.
48 P. J. Knowles and H. J. Werner, Chem. Phys. Lett., 1988, 145, 514-22.

49 E. R. Davidson, J. Comput. Phys., 1975, 17, 87-94.

50 S. R. Langhoff and E. R. Davidson, Int. J. Quantum Chem., 1974, 8, 61-72.

$51 M O L P R O$ is a package of $a b$ initio programs written by H.-J. Werner, P. J. Knowles, M. Schütz, R. Lindh, P. Celani, T. Korona, G. Rauhut, F. R. Manby, R. D. Amos, A. Bernhardsson, A. Berning, D. L. Cooper, M. J. O. Deegan, A. J. Dobbyn, F. Eckert, C. Hampel, G. Hetzer, A. W. Lloyd, S. J. McNicholas, W. Meyer, M. E. Mura, A. Nicklaß, P. Palmieri, R. Pitzer, U. Schumann, H. Stoll, A. J. Stone, R. Tarroni and T. Thorsteinsson.

52 J. R. McDonald, J. W. Rabalais and S. P. McGlynn, J. Chem. Phys., 1970, 52, 1332.

53 A GENERAL RRKM PROGRAM. L. Zhu, W. L. Hase, 1993. QCPE-644; Available from Quantum Chemistry Program Exchange http://www.qcpe.indiana.edu/. 